\title{
Fat Embolism Syndrome - A Rare Case of Bilateral Embolism of the Central Retinal Artery
}

Margarida Fonseca ${ }^{1 *}$, Dina Leal ${ }^{2}$, Cristina Freitas ${ }^{3}$, Bruno Gonçalves $^{4}$, Ricardo Alves $^{1}$, Maria Joao Silva $^{1}$, Sandra Guimaraes $^{3}$, José Eduardo Oliveira ${ }^{2}$ and Luís Lencastre ${ }^{2}$

${ }^{1}$ Internal Medicine Department, Hospital de Braga, Portugal

${ }^{2}$ Intensive Care Unit, Hospital de Braga, Portugal

${ }^{3}$ Ophthalmology Department, Hospital de Braga, Portugal

${ }^{4}$ Gastroenterology Department, Hospital de Braga, Portugal

\begin{abstract}
Fat embolism syndrome is a rare complication following a long bone fracture. It is believed to be caused by the toxic effects of free fatty acids and is clinically characterized by the triad of dyspnea, petechiae and mental confusion Ocular fat embolism syndrome in the complete absence of any cardiac defects is a rare phenomenon which is not commonly encountered in ophthalmic practice. The authors present a case of fat embolism syndrome with the uncommon finding of bilateral embolism of the central retinal artery after a single femoral fracture without evidence of cardiac defect.
\end{abstract}

Keywords: Fat embolism; Trauma; Purtscher's retinopathy; Foramen ovale; Intensive care

\section{Introduction}

Fat Embolism (FE) develops in $90 \%$ of all bone fractures. Fat Embolism Syndrome (FES) is a serious manifestation of FE with an incidence of $0.5-2.0 \%$ among all long bone fractures. It is believed to be caused by the toxic effects of free fatty acids but still, there is considerable controversy over both the source of fat emboli and their mode of action [1]. The exact pathogenesis is unclear but includes mechanical obstruction and biochemical injury.

Clinical diagnosis is the key because laboratory and imaging modalities (high-resolution computed tomography) are not specific. FES typically presents 24-72 hours after the initial injury. Patients present with a classic triad of respiratory changes, neurological abnormalities and petechial rash [2].

Purtscher described sudden blindness in patients with severe head trauma due to a remote retinopathy, characterized by bilateral retinal hemorrhages, cotton wool spots, and optic disc swelling seen on fundoscopy. A similar retinopathy has been reported in compressive chest trauma, long bone fractures, and acute pancreatitis. It is now well recognized that Purstcher's retinopathy can occur following bone fractures in association with FES [3,4].

Ocular fat embolism syndrome in the complete absence of any cardiac defects is a rare phenomenon which is not commonly encountered in ophthalmic practice [5]. The authors present a case of fat embolism syndrome with the uncommon finding of bilateral embolism of the central retinal artery after a single femoral fracture without evidence of any cardiac defect.

\section{Case Report}

A 19-year-old male without relevant past history was transferred to the emergency room after a car accident. The patient sustained a traumatic fracture of the right femoral shaft and had no evidence of thoracic, abdominal or head injury. He was hemodynamically stable, without any deterioration of consciousness and was admitted for surgical fixation. Five hours after admission he developed dyspnea and while awaiting surgery he suddenly developed self-limited seizure activity without subsequent recovery of consciousness. He was unresponsive to verbal stimuli, and no focal neurologic abnormalities were found on examination. He had a pulse rate of $132 \mathrm{bpm}$, blood pressure of $116 / 77 \mathrm{mmHg}$, respiratory rate of 32 breaths/min and oxygen saturation of $89 \%$, while breathing room air. Auscultation revealed normal heart sounds and fine diffuse inspiratory crackles. His Glasgow Coma Scale (GCS) score pointed 7 (E1V1M5). An arterial blood gas analysis revealed mild hypoxemia, but a radiograph of the chest was normal. Tracheal intubation was performed for airway protection. The blood test results were normal but because the D-dimer levels were elevated, a Computed Tomography (CT) scan was performed for better visualization of pulmonary vessels. Thoracic CT scan was normal and revealed no pulmonary emboli. For lack of improvement in level of consciousness, the patient also underwent cranial CT scan, which was normal. An electroencephalogram was performed and was normal. Less than twelve hours after admission, he developed widespread petechiae on the chest, axilla and conjunctiva (Figure 1) as well as respiratory failure, requiring mechanical ventilation. Electrocardiogram (ECG) revealed sinus tachycardia. The combination of long-bone fracture, petechial rash, hypoxemia and tachycardia within 24 hours after the accident prompted a diagnosis of FES. This patient was admitted to an Intensive Care Unit (ICU) and was managed conservatively till surgery. While in the ICU, he was referred to an ophthalmologist for dilated retinal examination in order to identify signs of Purstcher's

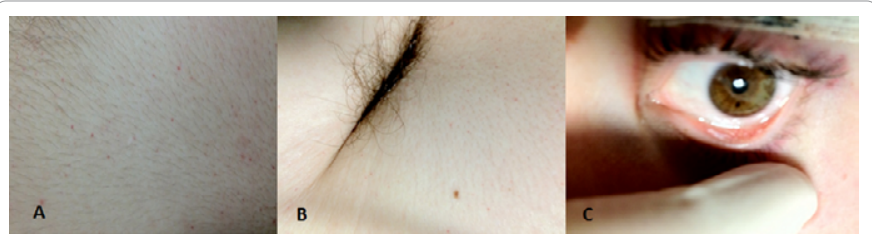

Figure 1: Fat embolism syndrome: petechial rash on the chest $(A)$, axilla $(B)$ and conjunctiva $(\mathrm{C})$.

*Corresponding author: Margarida Fonseca, Avenida de Sao Gonçalo 1350-3D to, 4835-105 Guimaraes, Portugal, Tel: +351 914620 337; E-mail: magutas@gmail.com

Received January 10, 2014; Accepted February 24, 2014; Published February 26, 2014

Citation: Fonseca M, Leal D, Freitas C, Gonçalves B, Alves R, et al. (2014) Fat Embolism Syndrome-A Rare Case of Bilateral Embolism of the Central Retinal Artery. J Clin Case Rep 4: 341. doi:10.4172/2165-7920.1000341

Copyright: (c 2014 Fonseca M, et al. This is an open-access article distributed under the terms of the Creative Commons Attribution License, which permits unrestricted use, distribution, and reproduction in any medium, provided the original author and source are credited. 
retinopathy. Fundoscopy showed Cotton-Wool Spots (CWS) along the major blood vessels and in the macular area, as well as hemorrhages in both eyes. These findings are consistent with bilateral embolization of central retinal artery. Echocardiography suggested normal cardiac function and revealed no signs of a patent foramen ovale or any other cardiac defect. With supportive treatment in ICU, hydration and enteral feeding, he gradually started to improve. On day three, he was submitted to surgery for fixation of femoral fracture and subsequently became arousable and begun to obey commands. He was extubated on day 11 and was transferred to the orthopedic ward on day 14, fully awake, obeying commands and hemodynamically stable. He was sent home ten days after discharge from the ICU. One year after the accident, he presented with 0.2 visual acuity in his right eye and 0.8 in his left eye, while bilateral CWS were still clearly visible on fundoscopy.

\section{Discussion}

Although its original clinical description dates from 1873 [6], fat embolism syndrome remains a diagnostic challenge for clinicians. Fat embolism is the presence of fat particles within the microcirculation, while FES is the systemic manifestation of fat emboli within the microcirculation. Common systemic manifestations include respiratory distress, altered mental status, and a rash. FES is most often associated with orthopedic trauma or surgery [7]. Rare cases of FES have been reported to occur following bone marrow transplantation, osteomyelitis, pancreatitis, alcoholic fatty liver, and even liposuction $[8,9]$.

There is considerable controversy over both the source of fat emboli and their mode of action. Two major theories have been proposed-the mechanical theory and biochemical one. According to the first theory, trauma to long bones releases fat droplets by disrupting fat cell in the fractured bone or in adipose tissue. These fat droplets enter the torn veins near long bone and are then transported to pulmonary vascular bed, where large fat globules result in mechanical obstruction and are trapped as emboli in the lung capillaries. Small fat droplets may pass through the lung and reach systemic circulation causing embolization to brain, skin, kidney or retina. Another way in which these fat droplets pass to the systemic circulation is pulmonary precapillary shunts and existing pathological venous-arterial communication such as patent foramen ovale (POF) $[10,11]$. This theory is supported by the description of "echogenic material" passing into the right heart during orthopedic surgery [12]. However, this theory does not sufficiently explain the 2472 hour delay in development after the acute injury. The second theory states that there is a number of biochemical mechanisms potentially involved in the development of FES. The most widely held is that the embolized fat is degraded in plasma to free fatty acids. Although neutral fat, such as is found in bone marrow, does not because an acute lung injury, it is hydrolyzed over the course of hours to several products, including free fatty acids, which have been shown to cause ARDS in animal models. Serum from acutely ill patients has been shown to have the capacity to agglutinate chylomicrons, low density lipoproteins, and liposomes of nutritional fat emulsions. The delay in development of symptoms could be explained by the time scale required to produce these toxic metabolites. The onset of symptoms may coincide with the agglutination and degradation of fat emboli. Levels of circulating free fatty acids are moderately elevated in fracture patients compared with controls. Nevertheless, evidence for these mechanisms of injury remains largely circumstantial $[2,8]$.

The fat embolism syndrome is characterized by an asymptomatic period of 24-72 hours (commonly 36 hours, although up to 6 days has been described) following bony injury or manipulation of the fracture site, and a symptomatic period which includes respiratory effects (95\%), cerebral effects (60\%) and petechiae (33\%) [13]. Our patient showed the classic triad of FES consisting of hypoxemia, neurological abnormalities, and petechial rash, less than 12hours before the accident. On searching medical literatures, we found only 4 patients who had latent period of less than 8 hours [14]. Tachycardia ( $>120$ per minute in up to $93 \%$ of cases), pyrexia $\left(>39^{\circ} \mathrm{C}\right.$ in up to $70 \%$ of cases), and pallor are also characteristic of this disorder. Since FES is a heterogeneous disease with no pathognomonic features, its diagnosis can be challenging. Gurd and Wilson proposed a combination of major and minor criteria for diagnosis of FES [15] (Table 1). Schonfeld has suggested a scoring system to help in diagnosis [16] (Table 2), while Lindeque proposed that FES can be diagnosed based on respiratory changes alone $[17,18]$ (Table 3 ). However, none of these criteria have been validated or have been universally accepted, and there is no pathognomonic test to confirm the diagnosis of the fat embolism syndrome which is usually one of exclusion [19]. Investigations are usually performed to corroborate the diagnosis or to monitor therapy [20]. Fundoscopy may reveal fat in the retinal vessels, fluffy exudates, hemorrhage and macular oedema. A severe form of retinal oedema known as Purtscher's retinopathy has also been described [4] and in the context of a traumatic event and the previously described triad of symptoms, makes the diagnosis of FES more consistent. According to our review, there are few cases of bilateral embolization of central retinal artery associated to FES in the absence of a cardiac defect. In

\begin{tabular}{|l|}
\hline MAJOR CRITERIA \\
\hline Petechial rash \\
\hline Respiratory insufficiency \\
\hline Cerebral symptoms in non-head injury patients \\
\hline MINOR CRITERIA \\
\hline Taquycardia $(>110 \mathrm{bpm})$ \\
\hline Fever $\left(>38.5^{\circ} \mathrm{C}\right)$ \\
\hline Retinal changes \\
\hline Jaundice \\
\hline Renal changes \\
\hline Thrombocytopenia \\
\hline Anaemia \\
\hline Elevated erythrocyte sedimentation rate \\
\hline Fat macroglobulinemia \\
\hline $\mathbf{2}$ major or $\mathbf{1}$ major and $\mathbf{4}$ minor criteria establishes the diagnosis of FES \\
\hline
\end{tabular}

Table 1: Gurd and Wilson's criteria for FES.

\begin{tabular}{|l|c|}
\hline CRITERIA & SCORE \\
\hline Petechial rash & 5 \\
\hline X-ray chest diffuse infiltrates & 4 \\
\hline Hypoxemia & 3 \\
\hline Fever & 1 \\
\hline Taquycardia & 1 \\
\hline Tachypnea & 1 \\
\hline Confusion & 1 \\
\hline Score $>\mathbf{5}$ diagnoses FES & \\
\hline
\end{tabular}

Table 2: Schonfeld's scoring system for FES.

CRITERIA

Sustained $\mathrm{pO}_{2}<8 \mathrm{kpa}$

Sustained $\mathrm{pCO}_{2}<7.3 \mathrm{kpa}$

Sustained respiratory rate $>35 / \mathrm{min}$, in spite of sedation

Increased work of breathing, dyspnea, tachycardia, anxiety

At least one of the above criteria diagnoses FES

Table 3: Lindeque's criteria for FES. 
this case, bilateral embolization of the central retinal artery occurred soon after a traumatic event, even though the presence of a POF was excluded, which could explain the short period of time until the development of symptoms as suggested by mechanical theory.

There is no specific therapy for the fat embolism syndrome, so prevention, early diagnosis, and adequate symptomatic treatment are of paramount importance [20,21]. Management of FES is primarily supportive including assisted ventilation and Intensive Care Unit (ICU) monitoring [22]. Early immobilization of fractures reduces the incidence of fat embolism syndrome and the risk is further reduced by surgical correction rather than conservative management. In our case, the patient had a seizure and was comatose 5 hours after the accident, which reinforces the need for early immobilization of fractures after a trauma event. Another strategy to prevent fat embolism syndrome is to limit the elevation in intraosseous pressure during orthopaedic procedures, in order to reduce the extravasation of intramedullary fat and other debris [21]. In a randomized trial of 40 patients, half were randomized to receive a venting hole for drainage of the medullary cavity between the greater and the lesser trochanter in order to limit intraoperative rises in intraosseous pressure. Significantly fewer major embolic events were detected by transoesophageal echocardiography in the venting group $(20 \%$ vs. $85 \%)$. Other operative refinements may also serve to limit intraosseous pressure, including the use of cementless fixation of hip prostheses and unreamed intramedullary femoral shaft stabilization [23]. Corticosteroid has been tried as both a therapeutic as well as a prophylactic agent in fat embolism syndrome. The role and efficacy of this hormonal product, in different forms and dosages, in diverse pulmonary complications have been debated by various researchers, with the potency proven beyond doubt in a few situations $[15,24,25]$. Despite the availability of multiple studies in the literature, the use of corticosteroid prophylaxis is controversial, largely because it is difficult to definitively prove efficacy in a condition with a low incidence, unclear risk factors, low mortality, and a good outcome with conservative management. Moreover, the lack of uniformity of the study designs and smaller sample sizes involved in all the above studies have further contributed to aggravating the ambiguity in this subject. Whether the corticosteroids can be routinely used as adjuvant drugs in addition to the existing protocol (and if used, in what dose and in which situations) to reduce the morbidity associated with FES is still an open question, and only a large confirmatory, multi-centered randomized controlled trial can provide the necessary evidence to guide patient care.

The mortality rate attributable to fat embolism ranges from $5-10 \%$ [26], with higher mortalities associated with fulminant fat embolism syndrome due to severe right heart failure, compared with the fat embolism syndrome in which the mortality relates largely to the mortality of the underlying respiratory failure or, rarely, with cerebral oedema [27]. The prognosis for patients who survive fat embolism is good, usually being complete within 2-4 weeks. In some cases permanent neurological disorders may persist. The visual changes are commonly reversible, although permanent changes in the retina and optic atrophy can occur [28], as we have seen in our patient.

\section{Conclusion}

Fat embolism syndrome is a rare complication occurring in 0.5 to $2 \%$ of patients following a long bone fracture. Because there is no pathognomonic test to confirm the diagnosis of the fat embolism syndrome, the diagnosis is usually one of exclusion and involves a high level of suspicion when dealing with a patient with respiratory difficulties, neurological impairment and petechiae. Ocular fat embolism syndrome in the complete absence of any cardiac defects is a rare phenomenon and there are few cases described in literature. Fundoscopy should be performed in order to identify and avoid permanent changes in visual acuity.

\section{References}

1. Sogunuru G, Moka N (2010) Fat embolism syndrome. Int J Emerg Med 3: 471

2. GuptaA, Reilly CS (2007) Fat embolism. Continuing Education in Anaesthesia, Critical Care \& Pain, p. 148-151.

3. Nayak H, Harun S, Palimar P (2005) Purtscher's retinopathy after fracture dislocation of shoulder joint. Emerg Med J 22: 831-832.

4. Roden D, Fitzpatrick G, O'Donoghue H, Phelan D (1989) Purtscher's retinopathy and fat embolism. Br J Ophthalmol 73: 677-679.

5. Nentwich MM, Remy M, Schaller UC (2011) Ocular fat embolism syndrome IntOphthalmol 31: 15-16.

6. Bergmann V (1873)Ein fall todlicherfettembolie. BerlKlinWchenschr.10:385.

7. Yeo SH, Chang HW, Sohn SI, Cho $\mathrm{CH}$, Bae KC (2013) Pulmonary and cerebral fat embolism syndrome after total knee replacement. J Clin Med Res 5: 239242.

8. Saigal R, Mittal M, Kansal A, Singh Y, Kolar PR, et al. (2008) Fat embolism syndrome. J Assoc Physicians India 56: 245-249.

9. Mellor A, Soni N (2001) Fat embolism. Anaesthesia 56: 145-154.

10. Pell AC, Hughes D, Keating J, Christie J, Busuttil A, et al. (1993) Brief report: fulminating fat embolism syndrome caused by paradoxical embolism through a patent foramen ovale. N Engl J Med 329: 926-929.

11. Watson AJ (1970) Genesis of fat emboli. J ClinPatholSuppl (R CollPathol) 4 132-142.

12. FortezaAM, Rabinstein A, Koch S, Zych G, Chandar J, et al. (2002) Endovascular closure of a patent foramen ovale in the fat embolism syndrome: changes in the embolic patterns as detected by transcranial Doppler. Arch Neurol 59: 455-459.

13. Bulger EM, Smith DG, Maier RV, Jurkovich GJ (1997) Fat embolism syndrome. A 10-year review. Arch Surg 132: 435-439.

14. Chen PC, Hsu CW, Liao WI, Chen YL, Ho CH, et al. (2013) Hyperacute cerebral fat embolism in a patient with femoral shaft fracture. Am J Emerg Med 31: 1420

15. Gurd AR, Wilson RI (1974) The fat embolism syndrome. J Bone Joint Surg Br 56B: 408-416.

16. Schonfeld SA, Ploysongsang Y, DiLisio R, Crissman JD, Miller E, et al. (1983) Fat embolism prophylaxis with corticosteroids. A prospective study in high-risk patients. Ann Intern Med 99: 438-443.

17. Lindeque BG, Schoeman HS, Dommisse GF, Boeyens MC, Vlok AL (1987) Fat embolism and the fat embolism syndrome. A double-blind therapeutic study. $J$ Bone Joint Surg Br 69: 128-131.

18. Vedrinne JM, Guillaume C, Gagnieu MC, Gratadour P, Fleuret C, et al. (1992) Bronchoalveolar lavage in trauma patients for diagnosis of fat embolism syndrome. Chest 102: 1323-1327.

19. Kwiatt ME, Seamon MJ (2013) Fat embolism syndrome. Int J CritlllnInjSci 3 64-68.

20. Glover P, Worthley LI (1999) Fat embolism. Crit Care Resusc 1: 276-284.

21. Mittal MK, Burrus TM, Campeau NG, Eckel LJ, Rabinstein AA, et al. (2013) Pearls \&oy-sters: good recovery following cerebral fat embolization with paroxysmal hyperactivity syndrome. Neurology 81: e107-109.

22. Eriksson EA, Schultz SE, Cohle SD, Post KW (2011) Cerebral fat embolism without intracardiac shunt: A novel presentation. J Emerg Trauma Shock 4 309-312.

23. Kim Y, Oh S, Kim J(2002) Prevalence of fat embolism following bilateral simultaneous and unilateral total hip arthroplasty performed with or without cement: a prospective, randomized clinical study. J Bone Joint Surg Am. 84A: 1372-1379.

24. Bederman SS, Bhandari M, McKee MD, Schemitsch EH (2009) Do corticosteroids reduce the risk of fat embolism syndrome in patients with longbone fractures? A meta-analysis. Can J Surg 52: 386-393. 
Citation: Fonseca M, Leal D, Freitas C, Gonçalves B, Alves R, et al. (2014) Fat Embolism Syndrome-A Rare Case of Bilateral Embolism of the Central Retinal Artery. J Clin Case Rep 4: 341. doi:10.4172/2165-7920.1000341

25. Sen RK, Tripathy SK, Krishnan V (2012) Role of corticosteroid as a prophylactic measure in fat embolism syndrome: a literature review. MusculoskeletSurg 96: $1-8$.

26. Fulde GW, Harrison P (1991) Fat embolism--a review. Arch Emerg Med 8: 233239
27. Etchells EE, Wong DT, Davidson G, Houston PL (1993) Fatal cerebral fat embolism associated with a patent foramen ovale. Chest 104: 962-963.

28. Peltier LF (1965) The Diagnosis of Fat Embolism. Surg Gynecol Obstet 121: 371-379. 